\title{
Wigner Crystals in the lowest Landau level at low filling factors
}

\author{
Kun Yang ${ }^{1}$, F. D. M. Haldane ${ }^{2}$, and E. H. Rezayi ${ }^{3}$ \\ ${ }^{1}$ National High Magnetic Field Laboratory and Department of Physics, Florida State University, Tallahassee, Florida 32306 \\ ${ }^{2}$ Department of Physics, Princeton University, Princeton, New Jersey 08544 \\ ${ }^{3}$ Department of Physics, California State University, Los Angeles, California 90032
}

(May 8, 2001)

\begin{abstract}
We report on results of finite-size numerical studies of partially filled lowest Landau level, at low electron filling factors. We find convincing evidence suggesting that electrons form Wigner Crystals at sufficiently low filling factors, and the critical filling factor is $\nu_{c} \approx 1 / 7$. At $\nu=1 / 7$ we find the system undergoes a phase transition from the Wigner Crystal to the incompressible Laughlin state when the short-range part of the Coulomb interaction is modified slightly. This transition is either continuous or very weakly first order.
\end{abstract}

73.20.Dx, 73.40.Kp, 73.50.Jt

Interacting electrons at sufficiently fow densities and temperatures were shown by Wigner to form a crystalline state. This ground state results from the dominance of the potential over the kinetic energy which occurs in the dilute limit. A Wigner Crystal (WC) state has been observed in a low-density two-dimensional (2D) electron gas system trapped on the surface of a helium liquid. 3 While unlikely to be observed at metallic densities in 3D this crystalline phase is in fact expected to occur in the $2 \mathrm{D}$ electron gas confined in semiconductor heterostructures or quantum wells, and subject to a strong perpendicular magnetic field (such that all electrons are in the lowest Landau level (LLL)). Under these conditions the kinetic energy is completely quenched making it an ideal environment in which to observe Wigner crystallization; the WC state is expected to have the lowest Coulomb interaction energy when the Landau level filling factor $(\nu)$ is sufficiently low. Indeed, substantial experimental evidence, mostly from transport measurements, points to a pinned WC state in the insulating phases of low $\nu$ However the situation is not yet definitive as direct experimental probe of the lattice structure of the $\mathrm{WC}$ is still lacking. In addition, the ubiquitous disorder in these systems could lead to other insulating phases such as the Hall insulator, or just complicate the detection of the crystal itself. On the theoretical side, early work 1 suggested that a $2 \mathrm{D}$ electron gas is always unstable against formation of a $\mathrm{WC}$ when subject to a strong magnetic field, at zero temperature. However, the discovery of fractional quantum Hall effect (FQHE) has led to the realization 0 that electrons can also form incompressible liquid states, especially at the primary sequence $\nu=1 / m$, where $m_{0}=3,5, \cdots$. By comparing the energy of the Laughlin state with that of a correlated WC state (whose energy is considerably lower than that of a simple Hartree-Fock estimate cluded that the critical filling factor $\nu_{c}$ below which the WC forms is slightly above $1 / 7$. This is in good agreement with transport experiments on clean samples. A more recent study using a formalism based on composite fermion 10 (CF) wavefunctions calculated the collective excitation energy variationally and found that the ground state become unstable against proliferation of magnetorotons (which points to formation of WC) for $\nu \leq 1 / 9$, although such an instability was not found at $\nu=1 / 7$. A similar trend was observed earlier in the single mode approximation 11 where the magneto-roton gap while not zero was found to be diminished considerably at $1 / 7$ and further at $1 / 9$. This was interpreted as a precursor to magneto-roton mode softening and the transition to the WC phase.

In this paper we present finite size exact diagonalization studies of systems with low $\nu$ which directly reveal the formation of the WC for the first time in the LLL. We study systems with torus geometry. Our previous work on charge density wave (CDW) ground states in high Landau level 12,13 has demonstrated that the torus geometry is advantageous as compared to, say, the spherical geometry 14, in studies of states with broken translational symmetry. The reasons are: (i) on a torus one can adjust the geometry to better accommodate a lattice in a finitesize system. (ii) The presence of translational_symmetry allows one to define a many-body momentum 15 that can be used to label the eigenstates. By inspecting the momenta of the low-lying states, as well as the wavevector dependence of the density-density correlation functions, one can easily detect whether the translational symmetry is broken in the system, and determine lattice structure of the broken symmetry (crystal) state.

We find strong evidence that WC forms at filling factors $\nu \leq 1 / 7$, and that the critical filling factor $\nu_{c}$ is very close to $1 / 7$. Furthermore, we have studied the competition between the WC state and the Laughlin state at $\nu=1 / 7$ by varying the geometries of our finite-size systems, as well as the short-range part of the Coulomb interaction. We find that the Laughlin state is always continuously deformed into one of the low-lying states that correspond to the WC phase, when the geometry or the interaction is varied. This suggests that the quantum phase transition between the incompressible FQHE and compressible $\mathrm{WC}$ phases is either continuous or very weakly first-order. The methods used here are similar to our previous studies and provide a reliable way of detecting broken symmetry in finite systems. We first investi- 
gate dependence of the spectrum on PBC geometry.
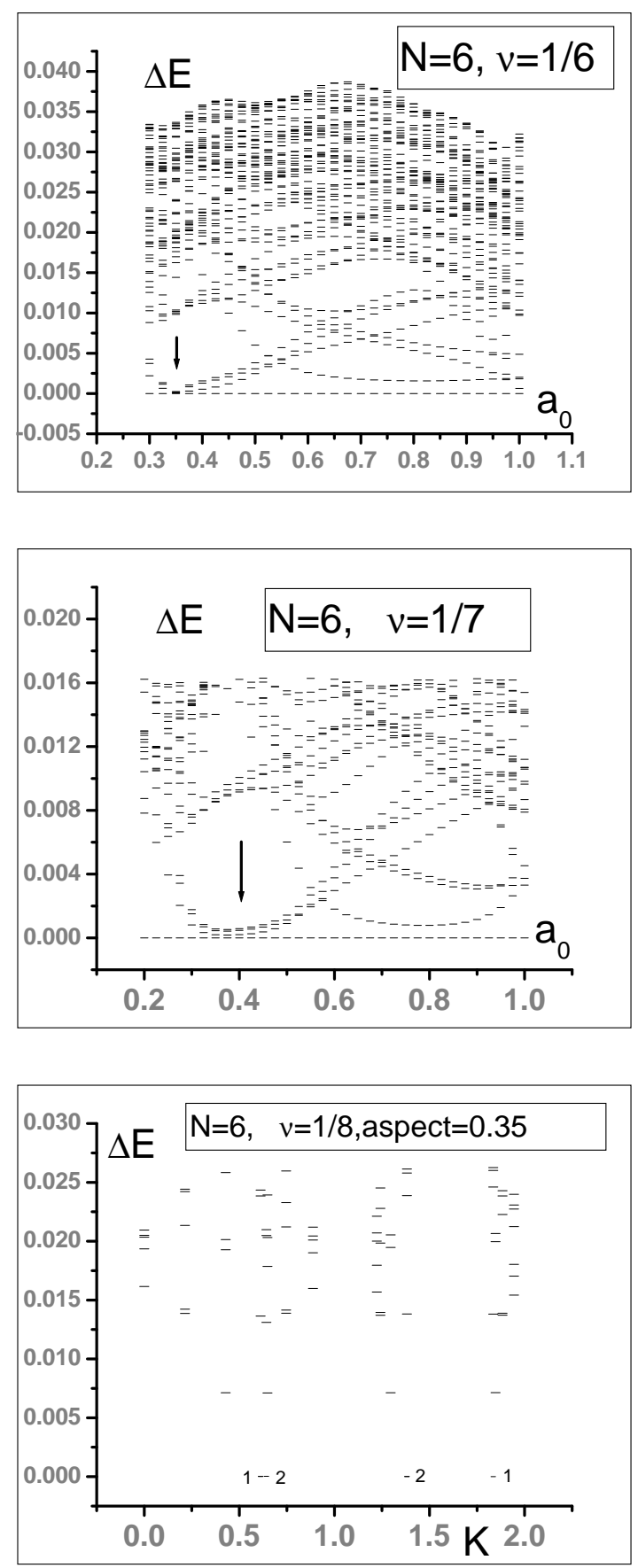

FIG. 1. The spectra of rectangular-shaped finite-size systems with $N=6$ electrons, at filling factors $1 / 6,1 / 7$, and $1 / 8$. The energy levels are plotted as functions of the aspect ratio for $\nu=1 / 6$ and $1 / 7$, and plotted as a function of the magnitude of momentum $\mathbf{K}$ for $\nu=1 / 8$, at aspect ratio 0.35 . The degeneracy of each low-lying level is indicated by the integer beside it for $\nu=1 / 8$.

Fig. 1 shows the energy spectra of systems with $N=6$ electrons, at filling factors $\nu=1 / 6,1 / 7$, and $1 / 8$, with rectangular geometry, for pure Coulomb interaction. For $\nu=1 / 6$ and $1 / 7$ we plot energy levels for a series of aspect ratio $a_{0}$, while for $\nu=1 / 8$ we plot the energy level versus the magnitude of many-body momentum $\mathbf{K}$, for $a_{0}=0.35$. One can see that for the $\nu=1 / 8$ case, as well as $\nu=1 / 7$ for a sizable range of $a_{0}: 0.3<a_{0}<0.5$, there are $N_{D}=6$ nearly degenerate low-lying states (including the ground state) that form what we call the ground state manifold. The K's of the states in this manifold form a $2 \mathrm{D}$ array, as we show in Fig. 2 for $\nu=1 / 7$ at $a_{0}=3 / 8$, indicating that the translational symmetry is broken in both directions, and the system has $2 \mathrm{D}$ crystalline order. These K's determine the lattice structure of the crystal. The number of unit cells included in the finite size system can be determined easily 13: $N_{c}=\bar{N}^{2} / N_{D}$, where $\bar{N}$ is the highest common divisor of $N$ and the number of flux quanta $N_{\phi}$. Here we have $\bar{N}=6$ in both cases, thus $N_{c}=6=N$, indicating there is one electron per unit cell. This is precisely what one expects for a Wigner Crystal (WC). This suggests that electrons form a WC at these filling factors, at least when a WC can be easily accommodated by the geometry of the finite size system.

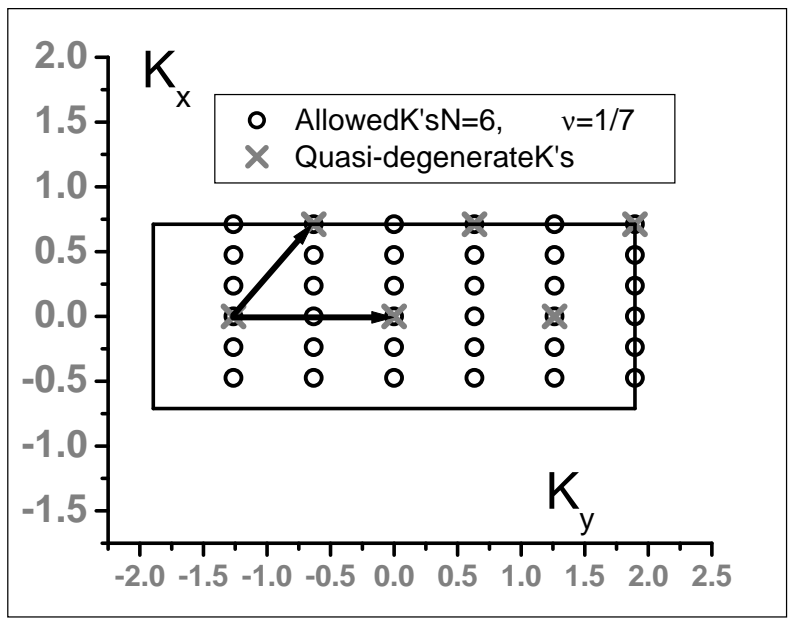

FIG. 2. The allowed momenta $\mathbf{K}$ for the $\nu=1 / 7$ system with $N=6$ electrons with aspect ration $a_{0}=3 / 8$, and the K's of the ground state manifold. These low-lying K's form a nearly triangular array, which represent the reciprocal lattice of the Wigner Crystal that the electrons form.

For $\nu=1 / 6$, on the other hand, such a (near) degeneracy is absent for almost all geometries, except for perhaps at $a_{0} \approx 0.35$. This suggests that the translational symmetry is not broken at $\nu=1 / 6$, although the tendency toward $\mathrm{WC}$ formation is present, especially when the geometry of the finite-size system is favorable. We thus conclude that the critical filling factor $\nu_{c}$ below which the Wigner Crystal forms is close to and probably slightly above $1 / 7$, which is in excellent agreement with the estimate of Lam 
and Girvin 8 ¿From the superlattice primitive basis vectors in the reciprocal space 13 we obtain the parameters of the WC unit cell $a_{1} \approx 4.421, a_{2} \approx 6.655$, and $\theta \approx 131.6^{\circ}$. While this is not exactly a triangular lattice it is close to it. We believe the discrepancy is caused by the finite size of the system, and in the thermodynamic limit we expect to recover a triangular lattice. We emphasize the fact that the WC state is stabilized near $a_{0}=0.4$ is not an artifact of the geometry of the finite size system; as we will see below at filling factors $\nu=1 / 3$ and $1 / 5$ where the system is an incompressible fluid and well described by the Laughlin state, no crystalline order is developed and the ground state has high overlap with the Laughlin state for all reasonable geometries, including the ones that are favorable for WC formation.

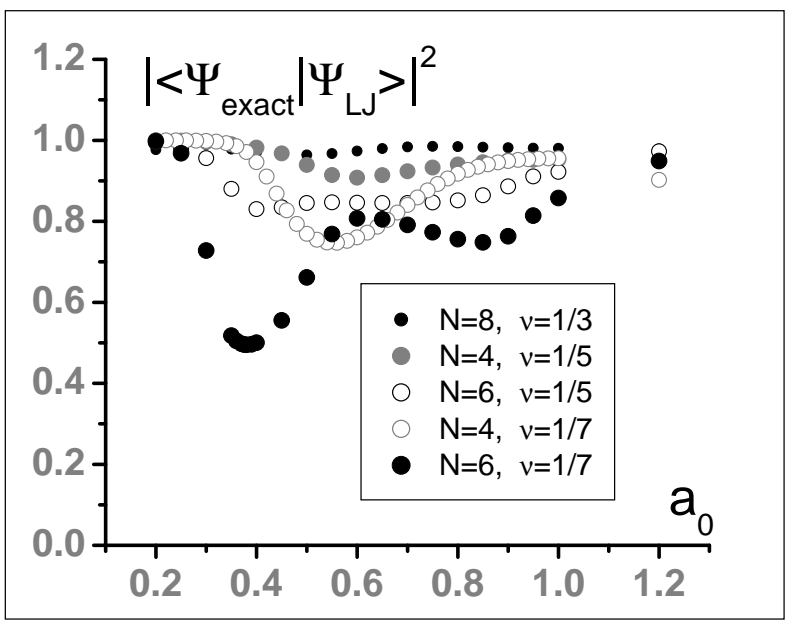

FIG. 3. The square of the overlap between the Laughlin state and the exact ground state of systems with rectangular geometry and a variety of filling factors and number of electrons, as a function of aspect ratio. The points at 1.2 are for hexagonal unit cells.

At $\nu=1 / m$ with $m$ being an odd integer, it is known 16 that for certain short-range repulsive interactions, the Laughlin state is the exact ground state that describes an incompressible fractional quantum Hall liquid. In Fig. 3 we show the square of the overlap between the Laughlin state with the exact ground state of the system for $m=3,5$ and 7 , at different aspect ratios and system sizes. It is seen that for $\nu=1 / 3$ and $1 / 5$, the overlap is rather insensitive to the geometry of the unit cell and is close to 1 , while for $\nu=1 / 7$ the square of the overlap varies appreciably with the geometry, and dips below 0.75 and 0.5 in certain range of $a_{0}$ for $N=4$ and $N=6$ respectively. The sensitivity of the energy spectrum as well as the overlaps on the system geometry is a strong indication that the ground state is compressible. It reflects the fact that the system wants to form a WC, but is frustrated when the aspect ratio $a_{0}$ is far from the optimal one for forming a crystal, $a_{0} \approx 0.4$ for $N=6$. It should be noted that the Laughlin state has $\mathbf{K}=0$, which is one of the $\mathbf{K}$ 's in the ground state manifold, and in the case of a pure Coulomb interaction the ground state turn out to be in the $\mathbf{K}=0$ sector for all the aspect ratios and sizes that we explored; thus the square of the overlap evolves continuously from being close to 1 near $a_{0}=1$, where the WC state is frustrated, to be less than 0.5 near $a_{0}=0.4$ where there is little geometric frustration for WC formation. Physically the reason that the overlap is large when the WC state is frustrated is that the Laughlin state at $\nu=1 / 7$, while describing an incompressible liquid state, already has substantial short-range crystal order built into it; it is thus energetically still competitive.

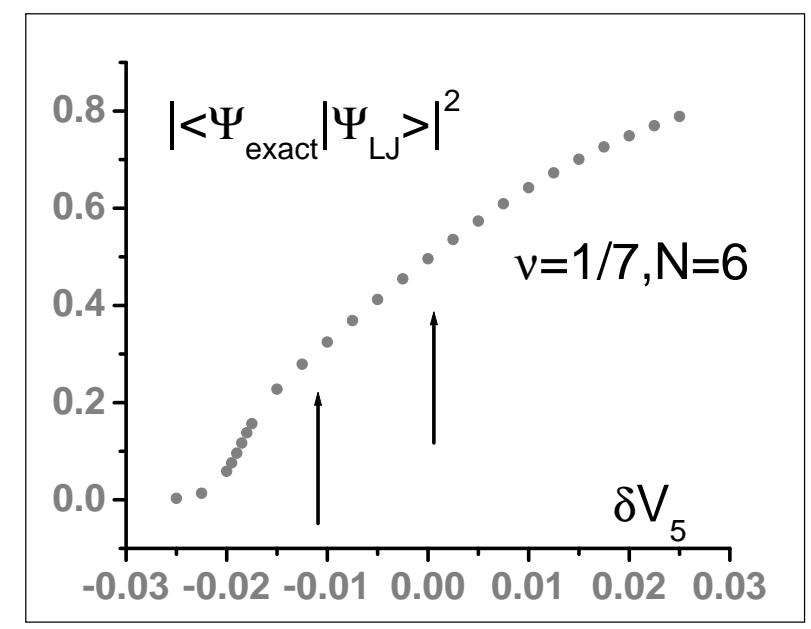

FIG. 4. The square of the overlap between the Laughlin state and the exact ground state, in a system with aspect ratio $a_{0}=3 / 8$. It is plotted as a function of $\delta V_{5}$ (see text). The arrow on the right hand side indicates the position at which the near-degeneracy of the low-lying states starts to develop. The arrow on the left hand side indicates the position of $\delta V_{5}$ below which the absolute ground state is not in the $K=$ sector. The overlap shown is between the Laughlin state and the lowest energy $K=0$ state.

At $\nu=1 / 7$, the Laughlin state can be stabilized by slightly modifying the short range part of the Coulomb potential. In Fig. 4 we plot the square of the overlap between the Laughlin state and the exact ground state of the system at $a_{0}=3 / 8$, as a function of $\delta V_{5}$, the change of the $m=5$ pseudo-potential1 6 from its Coulomb value. It is seen that the overlap is substantially improved to be above 0.8 by adding a small pseudopotential $\delta V_{5}=0.025 e^{2} / \epsilon \ell$ (which represents less than $10 \%$ increase of the $V_{5}$ pseudo-potential), even though the system geometry is most favorable for WC formation. This is further indication that $\nu=1 / 7$ is very close to the phase boundary separating the crystal and liquid phases. The smooth change of the overlap as a function of $\delta V_{5}$ also suggests the transition is either continuous or very weakly first-order in the thermodynamic limit. 
Adding a negative $\delta V_{5}$, on the other hand, further suppresses the overlap; in particular, at $\delta V_{5} \approx-0.011 e^{2} / \epsilon \ell$ there is a level crossing for the ground state, and for $\delta V_{5}<-0.011 e^{2} / \epsilon \ell$ the absolute ground state has a different wavevector from the Laughlin state; thus the overlap between the Laughlin state and the absolute ground state is strictly speaking zero here. However, the quasidegeneraces are well developed and the ground state is not unique. We have tracked the overlap with the $K=0$ "ground state". In real systems, the short-range part of the Coulomb interaction is softened due to the finite extent of the electron wave function along the $\hat{z}$ direction (or finite layer thickness), thus the WC state is expected to be further stabilized; we have studied potentials of typical layer thickness and find it is indeed the case at $\nu=1 / 7$.

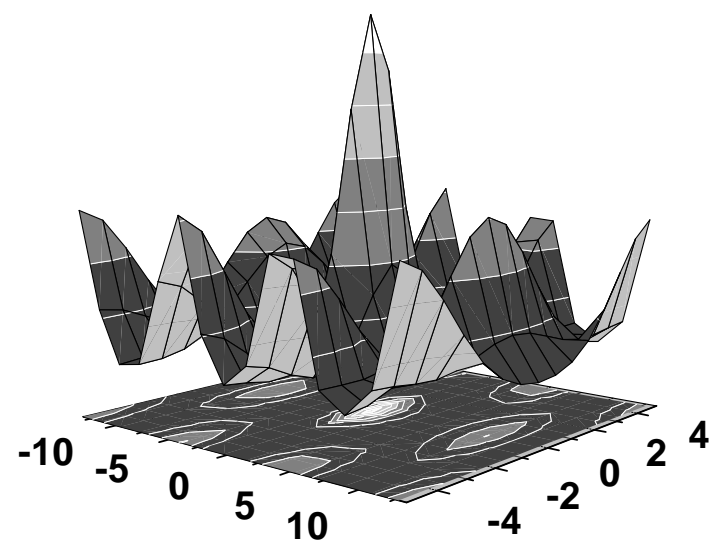

FIG. 5. The real-space lowest Landau level projected (or guiding center) density-density correlation function, for $N=6$ electrons at filling factor $1 / 7$, in a rectangular system with aspect ratio $3 / 8$. A $2 \mathrm{D}$ contour plot is also shown.

As in our previous studies we now turn to the density response functions. In Fig. 5 we show the LLL projected (or guiding center) density-density correlation function, in real space. This is the Fourior transform of the guiding center static structure factor 13 . The central peak represents the usual $\delta(\mathbf{r})$ singularity projected to the LLL, which is at the position of the electron where the correlation is measured from. The crystal structure can be clearly seen; the number of peaks (including the central one) equals the number of electrons $N=6$, indicating each unit cell includes one electron, which is what one expects for a Wigner Crystal.

The tendency toward WC formation, as well as the structure of the resultant lattice, can also be seen in the density response function in momentum space, $\chi(\mathbf{q})$. Fig. 6 is a plot of $\chi(\mathbf{q})$ for the same system as in Fig. 5. Here we see strong response at an array of momenta, which form a lattice that is close to being triangular. This is essentially the reciprocal lattice of the crystal that forms in real space. The origin of the large response at these momenta is the near-degeneracy of the low-lying states that are connected by these momenta discussed earlier; small energy denominators lead to large response at these momenta.

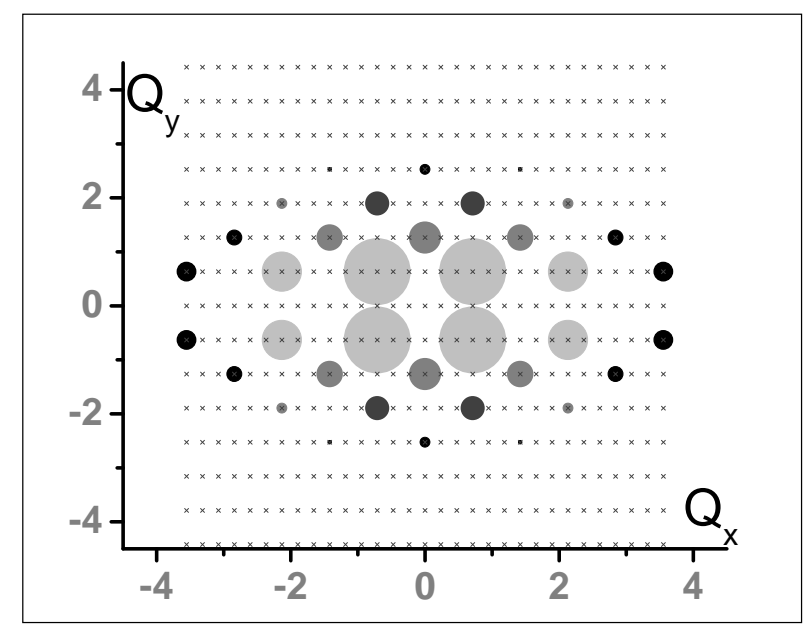

FIG. 6. A 2D plot of the LLL projected density response function $\chi(\mathbf{q})$, with the size of the dot representing the strength. The system is the same as that if Fig. 5. The 4 large dots represent a peak value of about 21000 . The nearby smaller dots along the $Q_{y}$ axis represent a value of 4700 etc.

In summary, we have shown that FQHE incompressible fluids become unstable around $\nu=1 / 7$ in agreement with previous predictions. We find the Wigner crystal becomes the ground state as the system geometry is adjusted to accommodate the crystal. It is more difficult to determine the order of the transition by the small sizes that we have studied. However, a strong level crossing first order transition seems to be ruled out by our study. We observe only a continuous evolution of the required quasi-degenerate manifold which always includes the state at $K=0$. This state for geometries that frustrate the crystal shows large overlap with the Laughlin's state. This suggest the transition may be second order but a weakly first order transition predicted by a mean field approach 11 also remains a possibility.

FDMH and EHR thank the Aspen Center for Physics where this work was started. EHR is grateful to NHMFL for their hospitality where it was brought to a conclusion. This work was supported by NSF grants DMR9809483 (FDMH), DMR-0086191 (EHR), DMR-9971541 $(\mathrm{KY})$, and the A. P. Sloan Foundation (KY). 
${ }^{1}$ E. P. Wigner, Phys. Rev. 46, 1002 (1934).

${ }^{2}$ C. C. Grimes and G. Adams, Phys. Rev. Lett. 42, 795 (1979).

${ }^{3}$ For reviews, see, e.g., chapters by H. Fertig and M. Shayegan in Perspectives in Quantum Hall Effects, edited by S. Das Sarma and A. Pinczuk, Wiley, New York (1997).

${ }^{4}$ H. Fukuyama, P. M. Platzman, and P. W. Anderson, Phys. Rev. B 19, 5211 (1979).

${ }^{5}$ R. B. Laughlin, Phys. Rev. Lett. 50, 1395 (1983).

${ }^{6}$ D. Yoshioka and H. Fukuyama, J. Phys. Soc. Jpn. 47, 394 (1979); D. Yoshioka and P. A. Lee, Phys. Rev. B 27, 4986 (1983).

${ }^{7}$ K. Maki and X. Zotos, Phys. Rev. B 28, 4349 (1983).

${ }^{8}$ P. K. Lam and S. M. Girvin, Phys. Rev. B 30, 473 (1984).

${ }^{9}$ R. K. Kamilla and J. K. Jain, Phys. Rev. B 55, R13417 (1997).

10 J. K. Jain, Phys. Rev. Lett. 63, 199 (1989).

${ }^{11}$ S. M. Girvin, A. H. MacDonald, and P. M. Platzman, Phys. Rev. B 33, 2481 (1986).

${ }^{12}$ E. H. Rezayi, F. D. M. Haldane, and K. Yang, Phys. Rev. Lett. 83, 1219 (1999).

${ }^{13}$ F. D. M. Haldane, E. H. Rezayi, and K. Yang, Phys. Rev. Lett. 85, 5396 (2000).

${ }^{14}$ F. D. M. Haldane, Phys. Rev. Lett. 51, 605 (1983).

${ }^{15}$ F. D. M. Haldane, Phys. Rev. Lett. 55, 1095 (1985).

${ }^{16}$ F. D. M. Haldane, in The Quantum Hall Effect, 2nd Ed., edited by R. E. Prange and S. M. Girvin (Springer, New York, 1990). 